\title{
Universal Scaling Laws for Homogeneous Dislocation Nucleation during Nano-Indentation
}

\author{
Akanksha Garg ${ }^{\mathrm{a}}$, Craig E. Maloney \\ Carnegie Mellon University \\ Pittsburgh, PA 15213
}

We perform atomistic simulations to study the mechanism of homogeneous dislocation nucleation in two dimensional (2D) hexagonal crystals during nanoindentation with a circular indenter of radius $R$. We study both a realistic embedded atom method (EAM) potential for $\mathrm{Al}$ in addition to simple pair-wise potentials: Lennard-Jones, Morse, and Hookean springs. The nucleation process is governed by the vanishing of the energy associated with a single energy eigenmode. The critical eigenmode, or dislocation embryo, is found to be localized along a line (or plane in 3D) of atoms with a lateral extent, $\xi$, at some depth, $Y^{*}$, below the surface. For all interatomic potentials, the scaled critical load, $F_{c} / R$, and scaled critical contact length, $C_{c} / R$, decrease to R-independent values in the limit of large $R$. However, $\xi / R$ and $Y^{*} / R$ display non-trivial scaling with $R$ despite the $R$ independence of $F_{c} / R$ and $C_{c} / R$. We show that although both the interaction potential and the orientation of the lattice affect the prefactors in the scaling relations, all the scaling laws are robust. Furthermore, we show that a stability criterion proposed by vanVliet et. al. based on the minimum eigenvalue, $\Lambda$, of the local acoustic tensor predicts the location, orientation, and polarization of the dislocation embryo with a high degree of accuracy for all potentials and crystallographic orientations. However, we also show that, for all crystallographic orientations and interaction potentials, $\Lambda$ erroneously indicates instability before the true instability occurs.

\footnotetext{
${ }^{a}$ Present address: FM Global. Norwood, MA 02135

b Present address: Northeastern University. Boston, MA 02115
} 


\section{INTRODUCTION}

The mechanical response of materials at the nanoscale can be dramatically different than the macroscopic response. Studying materials at the nanoscale is important both for providing the fundamental building blocks for understanding the macroscopic material response and for understanding the performance of emerging nanoscale devices. Recent work has focused on several different single crystal systems, both metallic and semiconductor, in various loading geometries such as micro- and nano-pillar compression ${ }^{1}$, nano-particlecompression $\left[56\right.$, and nano-indentation ${ }^{7}[17$. In these scenarios, one is typically interested in how temperature, loading rate, or the sample or probe size affects the mechanical response.

In this work, we focus on nanoindentation. Indentation geometries generally fall into one of two categories: sharp (like a rectangular punch) $\underline{18}$ or smooth indenters (like a cylindrical or spherical punch) $\frac{10}{10}$. In the former case of a sharp punch, there is no length scale in the problem beyond the lattice constant. In this case, the nucleation process has been shown 18 to be well described by the Peierls-Nabrarro-Rice ${ }^{19[20}$ continuum dislocation model. However, in the case of a smooth indenter, the radius of curvature of the indenter may enter the problem - indeed, we show below it does so in a dramatic way - and the situation is far less well understood.

Indentation experiments using smooth indenters on "real" metallic systems 21 and " analog" systems such as bubble rafts ${ }^{22}$, and colloidal crystals ${ }^{15}$ all provide robust evidence for subsurface nucleation events in the bulk, in agreement with earlier atomistic simulations.

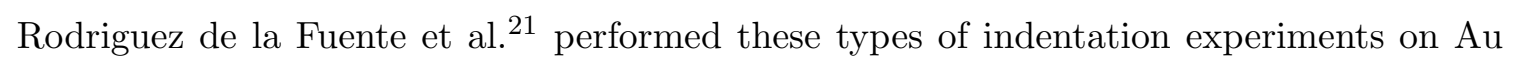
using an STM tip as an indenter and showed subsurface nucleation of dislocations and a corresponding pattern of complex surface steps. Gouldstone et. al.$^{22}$ also showed homogeneous dislocation nucleation events in 2D bubble raft experiments; macroscopic analogs of metallic systems. Schall et. al. used another analog system: a colloidal crystal, and observed thermally activated loop nucleation under the indenter tip. Kelchner et. al.9 observed subsurface dislocation nucleation in atomistic simulations during the indentation 
of $\mathrm{Au}$.

For a smooth indenter, the question of where in the sample and under what conditions dislocations nucleate is still surprisingly contentious. It is not even clear whether dislocation nucleation should be understood as a local material instability as in the case of a pre-existing dislocation reaching its Peierls threshold or a non-local instability as in the case of the Euler bucking of a column where the global loading geometry is crucial. Several attempts have been made at trying to understand the instability in terms of a local material threshold. The most naive approach associates instability with the local resolved shear stress exceeding some threshold value. Kelchner et. al. showed long ago that this naive approach fails completely ${ }^{9}$. The location of maximum local resolved shear stress was found to be far from the nucleation event. In a series of papers, vanVliet ${ }^{23}$, Zhu ${ }^{24}$, $\mathrm{Li} 25$, and co-workers put forward an approach based on the lowest eigenvalue, $\Lambda_{\min }$, of the atomic-level acoustic tensor $\frac{24,25}{2}$. They showed that the location of the zeros of $\Lambda$ could be used to identify and predict dislocation nucleation 23 , supporting the point of view that dislocation nucleation was essentially a local, material type instability. More recently, however, this point of view has been called into question. Miller and Rodney ${ }^{26}$ showed that $\Lambda$ could be less than zero in rather large regions of the crystal before the onset of instability. Furthermore they showed that in certain crystallographic orientations, the most negative value of $\Lambda$ was in a location far from the location of the dislocation nucleation event. To complicate the issue further, they showed clear evidence that the characteristic length of the critical eigenmode associated with the elastic instability had a dramatic dependence on the curvature of the indenter. This highlighted the non-local nature of the instability and further called into question the tenability of a local threshold condition and implied that the geometrical nature of the instability could not be neglected. These two issues, the way in which the bifurcation event scales with the indenter radius and the robustness of the $\Lambda$ criterion, are the topics of this paper.

In this work, we focus on the initial elastic bifurcation event and ignore subsequent 
plastic activity. This subsequent plastic activity is crucial for determining the size of the load drop associated with the so-called "pop-in" event, but should not have any impact on the value of the load at which that initial pop-in is observed. In particular, we focus on the spatial structure of the critical eigenmode responsible for the instability. We refer to it as the dislocation embryo. We study the scaling with indenter radius, $R$, of various quantities at the point of instability. These quantities include mechanical parameters - load, and nominal contact length - as well as geometrical characteristics of the embryo itself (depth of the embryo from surface, $Y^{*}$, and length of the embryo, $\xi$ ). Here $F_{c}$ and $C_{c}$ are the indenter load and nominal contact length at the onset of instability. $F_{c} / R$ represents the hardness in a $2 \mathrm{D}$ system. ${ }^{27}$ In the continuum limit for large values of $R, F_{c} / R$ and $C_{c} / R$ go to $R$ independent asymptotes as one would naively expect on dimensional grounds. However, $\xi$ and $Y^{*}$ exhibit non-trivial scalings with $R: \xi \sim R^{1 / 2}$ and $Y^{*} \sim R^{3 / 4}$. These scalings for the size and location of the embryo are completely inconsistent with a scale-free continuum description such as Cauchy-Born elasticity ${ }^{28}$ since the lattice constant must enter the analysis (on purely dimensional grounds). They indicate a complex interplay between the discrete lattice effects and the strain gradients induced by the indentation.

Furthermore, we perform a detailed analysis of the acoustic tensor field. Our results indicate that $\Lambda_{\min }$ becomes negative before the onset of instability, in agreement with Miller and Rodney 26. However, given that the system is driven precisely to the point of instability, the $\Lambda_{\min }$ field is smallest (most negative) at the center of the dislocation embryo and provides an excellent prediction of where in the crystal the embryo forms, in agreement with vanVliet et. al. ${ }^{23}$. This is true for all interaction potentials and all crystallographic orientations. We also show that the atom-wise $\Lambda$ tensor contains high fidelity information about the polarization and orientation of the embryo. 


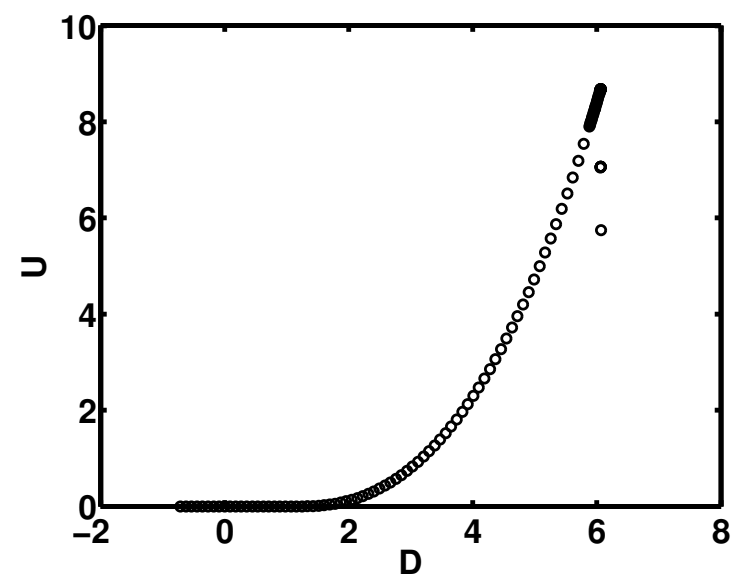

(a)

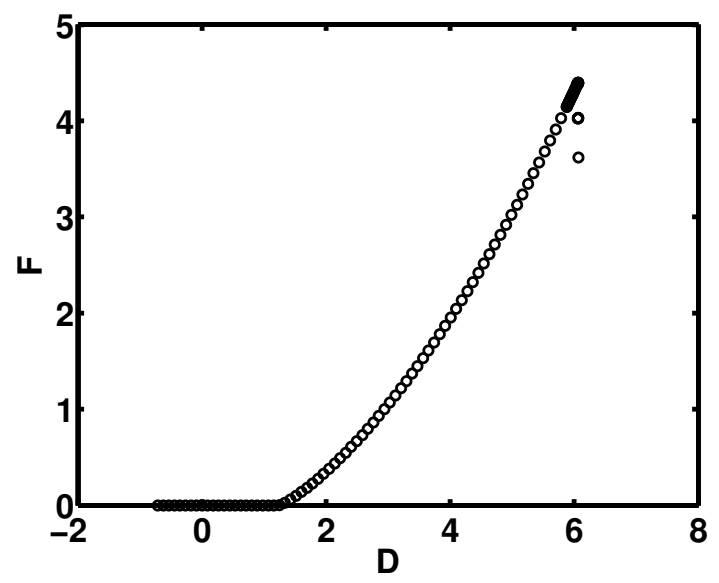

(b)

FIG. 1. (a) Elastic energy, $U$, stored in the crystal as a function of indenter depth, $D$, LJ crystal, $L=40, R=40$. (b) Corresponding load, $F$, on the indenter in the vertical direction as function of indenter depth, $D$.

\section{SIMULATION FORMALISM}

We perform athermal quasi-static nano-indentation simulations for 2D hexagonal crystals via energy minimization dynamics. The LAMMPS molecular dynamics framework ${ }^{29}$ is used to perform non-linear energy minimization with the Conjugate-gradient algorithm with the Polak-Ribere modification, and a custom python code was developed to perform eigenmode analysis using the sparse matrix routines in $\mathrm{SciPy}$. The resulting loaddisplacement and elastic energy-displacement curves for a typical indentation process are shown in Fig. 1. The force on the indenter and total potential energy in the crystal increase as the indenter moves into the crystal until the crystal becomes unstable. At the point of instability, the load and potential energy each undergo a discrete, discontinuous drop. The load drop is accompanied by the nucleation of a dislocation dipole as in Fig. 5(a). In this work, we are interested in the structure of the unstable mode precisely at the onset of instability. To reach as close as numerically possible to the nucleation event, 


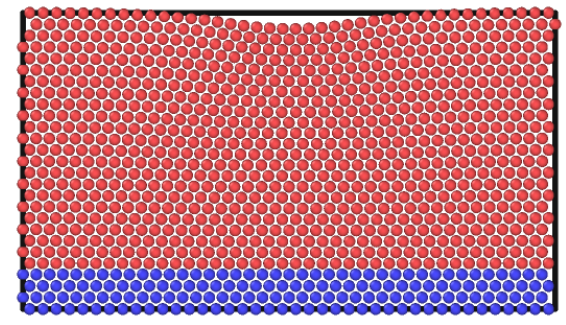

(a)

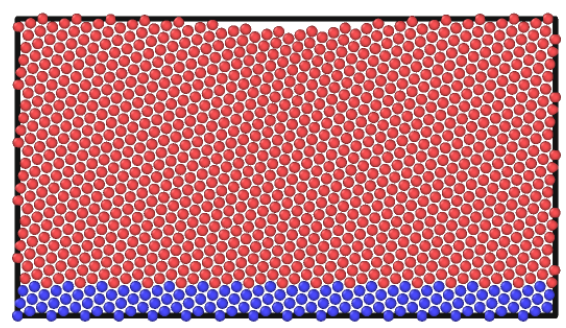

(c)

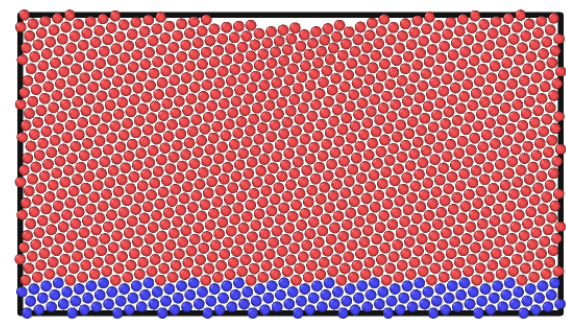

(b)

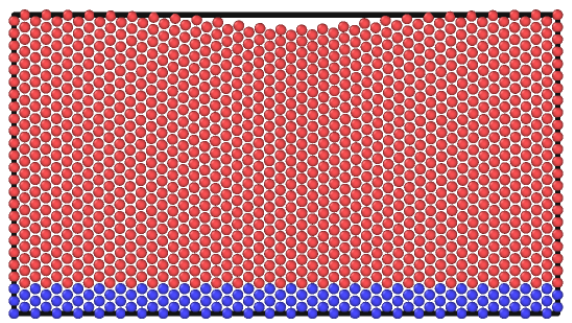

(d)

FIG. 2. Schematic of 4 different orientations of crystal with respect to indenter axis. The red atoms correspond to the crystal and the blue atoms correspond to the rigid base a) $\mathrm{O}_{1}$ b) $\mathrm{O}_{2}$ c) $\mathrm{O}_{3}$ d) $\mathrm{O}_{4}$

we use a dynamic indenter stepping algorithm similar to the algorithm used by $\mathrm{MR}^{26 \mid 31}$. When nucleation occurs, we return the indenter to its last stable position before nucleation, reduce the step size by a factor of 10 and restart our simulation.

We configure 2D hexagonal crystals as shown in Fig. 2 and 3 with periodic boundaries on the sides, rigid base at the bottom, and a circular indenter on top of the crystal. The geometrical parameters are shown in the setup diagram in Fig.3. We chose wide enough $L_{x}$ that our results are do not depend on it. In the rest of the document, all lengths such as: $L_{x}, L, R$ and $C$ are measured in units of the lattice constant, a. Energy is measured in units of $E a^{2}$ where $E$ is the two dimensional Young's Modulus for each interatomic potential on the hexagonal lattice.

We use a stiff, featureless, harmonic, repulsive, cylindrical indenter for all our simula- 


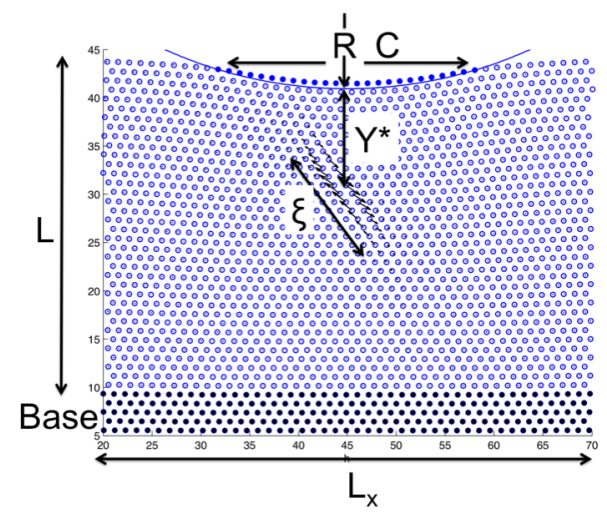

(a)

FIG. 3. Schematic of the setup. All the relevant geometrical parameters are shown: width, $L_{x}$; thickness, $L$; indenter radius, $R$; contact length, $C$. We indicate the crystallographic orientation with respect to indentation axis as: $O_{1}, O_{2}, O_{3}$, and $O_{4}$ respectively.

tions as in $(32,26)$. The interaction potential used to model the interaction between the indenter and the atoms is of the form:

$$
\begin{aligned}
\phi\left(r_{i}\right)=A\left(R-r_{i}\right)^{2} & \text { if } r_{i} \leq R, \\
0 & \text { if } r_{i}>R .
\end{aligned}
$$

Here $R$ is the radius of the indenter and $r$ is the distance of the particle from the indenter center. We note that this model for the interaction of an indenter with the sample is appropriate only for an indenter which is very stiff compared to the crystal, and the finite compliance used here and in related studies is simply a numerical convenience. We choose $A$ to be large enough that it does not affect any results but small enough that it does not cause numerical difficulties during the energy minimization procedure.

We study four different crystallographic orientations which we label: $O_{1}, O_{2}, O_{3}, O_{4}$ as shown in Fig. 2, $O_{1}$ has the nearest neighbor axis aligned normal to the indenter motion axis. In $O_{2}$ and $O_{3}$ the nearest neighbor axes are roughly aligned at $14^{\circ}$ and $19^{\circ}$ respectively from the indentation direction. In $O_{4}$ the nearest neighbor axis is parallel to the indentation direction. Note that the surfaces of $\mathrm{O}_{2}, \mathrm{O}_{3}$ and $\mathrm{O}_{4}$ are much rougher than 
the surface of $O_{1}$. To study the effect of the form of the interatomic interactions on the nucleation process we use 4 different potentials: Lennard-Jones (LJ), Morse, Harmonic (or Hookean), and an EAM potential. In the LJ and Harmonic potentials, there are no free parameter. For Morse, the exponential coefficient, $\alpha$, is 10 in our simulations. We use the Ercolessi-Adams EAM potential for $\mathrm{Al}$. 33 .

\section{KINEMATIC DESCRIPTION OF DISLOCATION NUCLEATION}

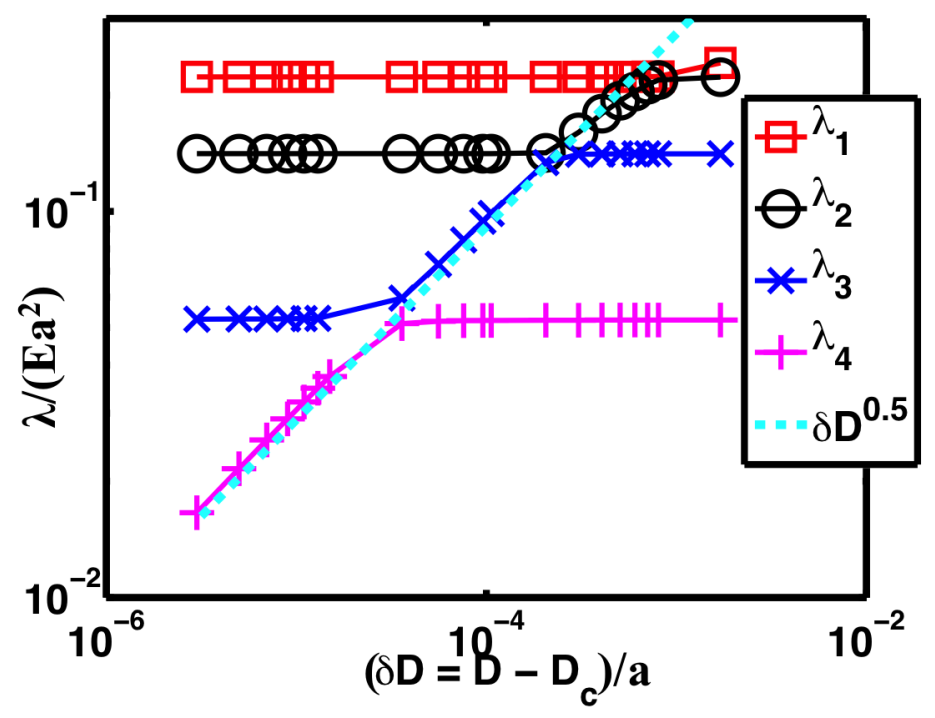

(a)

FIG. 4. Lowest 4 energy eigenvalues, $\lambda$, for $L=40, R=40, O_{1}$, LJ crystal, as function of $\delta D=D-D_{c}$. The cyan (dashed) line represents $\lambda \sim(\delta D)^{0.5}$.

The total potential energy, $U\left(x_{i \alpha}, D\right)$, is a function of atomic positions, $x_{i \alpha}$, and indenter depth, $D$. The first derivative of energy with respect to the particle position gives the force, $F_{i \alpha}$, on each particle as:

$$
F_{i \alpha}=-\frac{\partial U}{\partial x_{i \alpha}}
$$




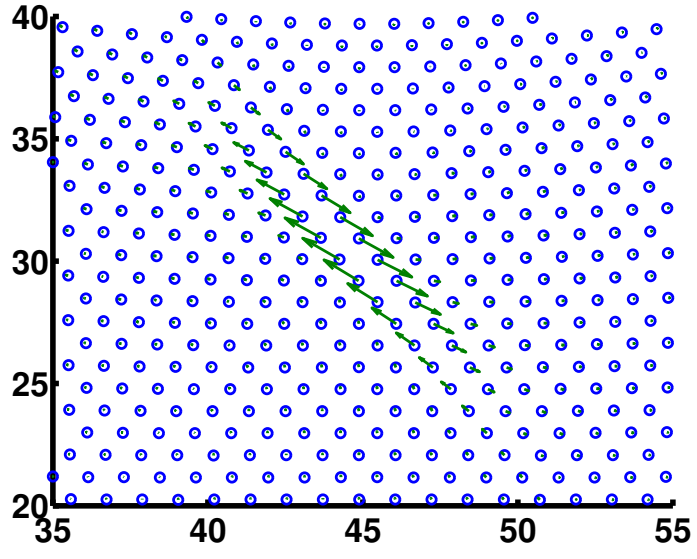

(a)

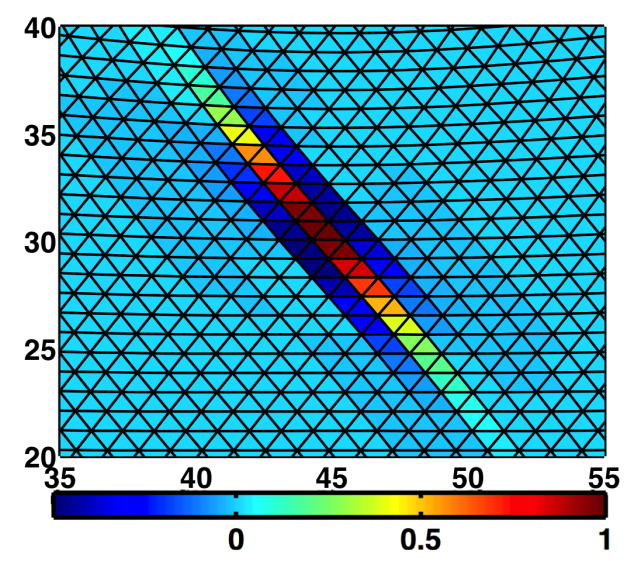

(b)

FIG. 5. (a) Lowest eigenmode at $\delta D=D-D_{c} \approx 10^{-6}$ for $L=40, R=40, O_{1}$, LJ crystal.

(b) Corresponding transverse mode gradient, $\Omega / \Omega_{\max }$.

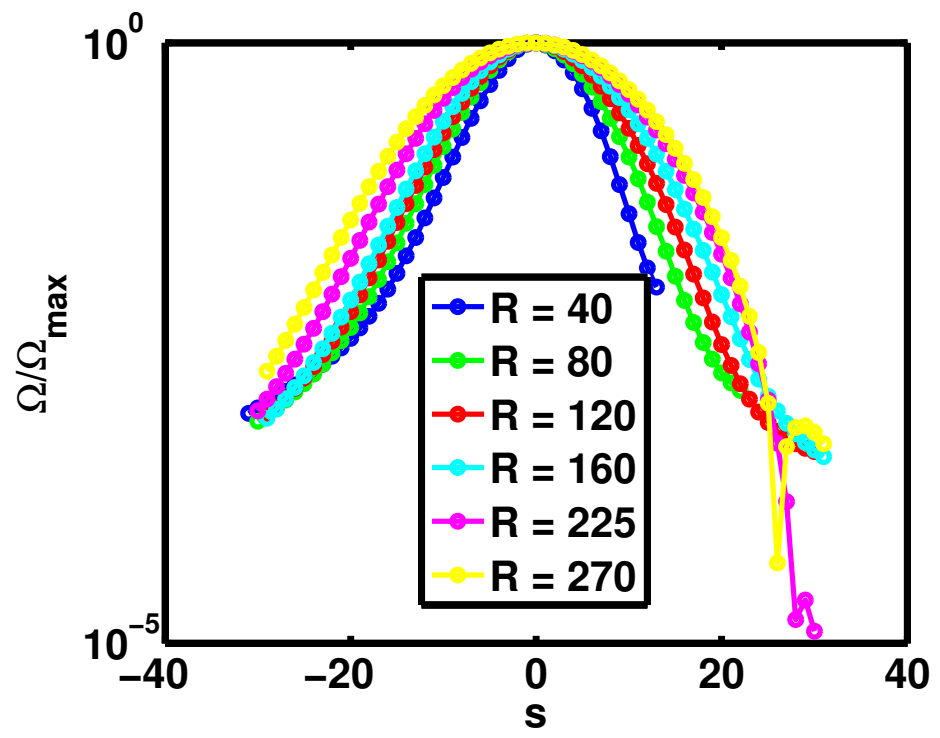

(a)

FIG. 6. Transverse mode gradient, $\Omega / \Omega_{\max }$, vs. distance, $s$, along the embryo for $L=80$, O1, LJ crystal.

Latin characters are used to index particle number, and Greek characters to index Cartesian 
components. Then, we use the Lanczos algorithm, as implemented in the SciPy toolkit ${ }^{30}$, to compute the lowest 4 eigenvalues of the Hessian matrix for the relaxed configuration at each indenter step. The Hessian matrix, $H_{i \alpha j \beta}$, is the second derivative of total potential energy as shown:

$$
H_{i \alpha j \beta}=\frac{\partial^{2} U}{\partial x_{i \alpha} \partial x_{j \beta}}
$$

The forces induced by an infinitesimal external indenter motion, $\Xi_{i \alpha}$, must be balanced by the internal atomic rearrangements as shown in $(\underline{34}, 35)$ : .

$$
\begin{gathered}
\Xi_{i \alpha}=-\frac{\partial F_{i \alpha}}{\partial D} \\
H_{i \alpha j \beta} \dot{x}_{j \beta}=\Xi_{i \alpha}
\end{gathered}
$$

Here, $\dot{x} \doteq \frac{d x}{d D}$. We refer to the derivative of the particle positions with respect to indenter depth as velocities since $D$ plays the role of time in quasi static indentation. We may solve (4) at each indentation step to compute the atomic velocities. The analytical expression of $H_{i \alpha j \beta}$ can be simply derived for pair potentials such as LJ potential and Morse Potential using 53

$$
M_{i \alpha j \beta}=\left(c_{i j}-\frac{t_{i j}}{r_{i j}}\right) n_{i j \alpha} n_{i j \beta}+\frac{t_{i j}}{r_{i j}} \delta_{\alpha \beta}
$$

where $t$ and $c$ are the first and second derivatives of the bond energy and $n_{i j \alpha}$ is the unit normal pointing from particle $i$ to particle $j$. Then, $H_{i \alpha j \beta}=-M_{i \alpha j \beta}$ for off diagonal terms and $H_{i \alpha i \beta}=\sum_{j} M_{i \alpha j \beta}$ for diagonal terms. However, for multibody potentials like EAM potential the calculation of Hessian matrix is more involved.

We compute the lowest 4 eigen values of the Hessian matrix for the relaxed configurations at each indenter step. In all cases, we observe that the system is driven to instability along a single eigenmode. This mode shows anti-parallel motion of small number of atoms on adjacent crystal planes as shown in fig. 5(a). This ultimately results in the homogeneous nucleation of a dislocation dipole. We denote the critical depth, $D_{c}$, as the depth of the indenter at which the dislocation dipole nucleates. Then, $\delta D=D-D_{c}$ is the distance of 
the indenter from the critical depth. It was shown earlier by Hasan and Maloney ${ }^{34}$ that in this case of dislocation nucleation, the bifurcation mechanism is saddle-node bifurcation. In a saddle-node bifurcation, a single eigenmode descends through the spectrum, and its eigen value vanishes as the square root of the distance to the dislocation nucleation. We call the energy-eigenmode corresponding to the eigen-value vanishing as $\delta D^{0.5}$, the critical mode. In fig. 4 the critical eigenvalue corresponds to the cyan line.

To calculate the precise location and size of the embryo we define a scalar quantity $\Omega$. For a given vector field, $\Omega$, is the transverse derivative of the vector field along a particular crystal axis in the deformed configuration. The lattice is first triangulated, and on each triangle, $\Omega$ is computed by linear interpolation of the critical mode vectors. Fig. $5(\mathrm{~b})$, shows $\Omega$ corresponding to the mode shown in Fig. 5(a). We define the center of the embryo to be at the centroid of the triangle with maximum $\Omega . s$ is defined as the distance of each triangle from the triangle corresponding to the maximum $\Omega$ along the crystal axis of interest. Fig. 6 shows $\Omega(s)$ for $L=80$ at various $R$ for $L J$ in orientation $O_{1}$. Note that the width of these $\Omega$ profiles increases with indenter radius and have a roughly Gaussian form near the embryo center. To define the embryo size, these $\Omega(s)$ profiles are fit to Gaussian functions of the form:

$$
\Omega(s) / \Omega_{\max }=e^{-s^{2} / \xi^{2}}
$$

We fit the $\log \Omega(s)$ curves out to their half-maximum using the generalized least square method to define, $\xi$, the embryo size. It is important to note that we look at configurations just before nucleation. The strain, although large, is quite uniform in the vicinity of the embryo, and these atomic configurations would have almost perfect centrosymmetry ${ }^{9}$ and almost vanishing slip vector $\frac{37}{2}$. $\Omega$ is roughly analogous to a slipping rate rather than a slip. 


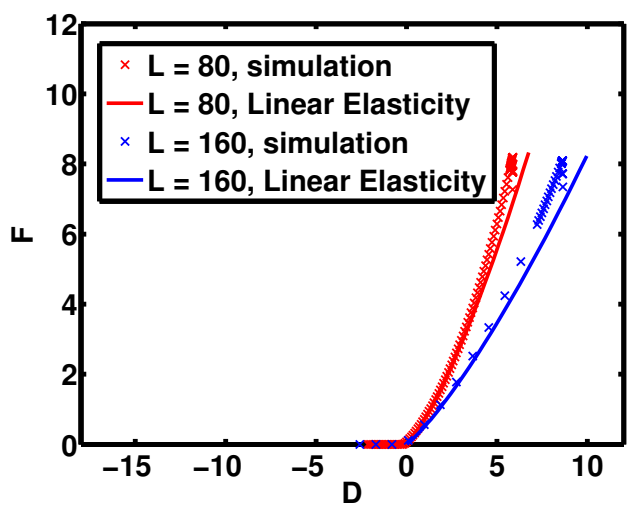

(a)

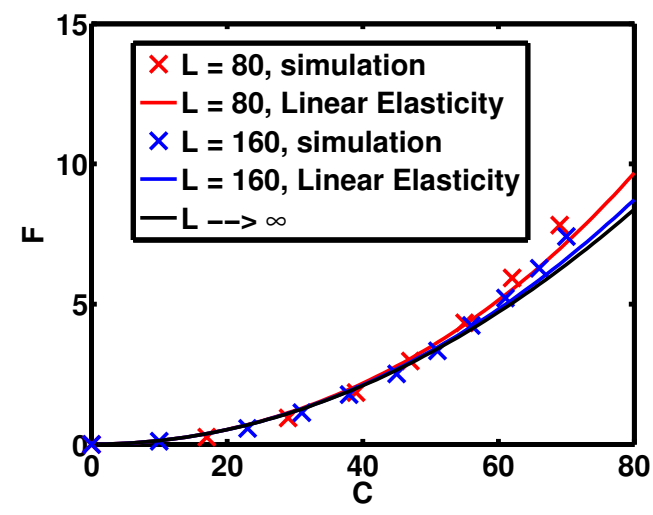

(b)

FIG. 7. (a) Indenter load, $F$, as a function of contact length, $C$ (b) Indenter load, $F$, as a function of indenter depth, $D$ for various $L, R=160, O_{1}$, LJ crystal. Solid lines are the predictions of Hertzian contact theory given by (7) and (8)

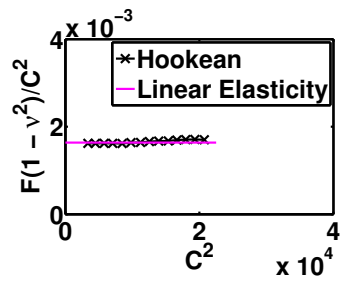

(a)

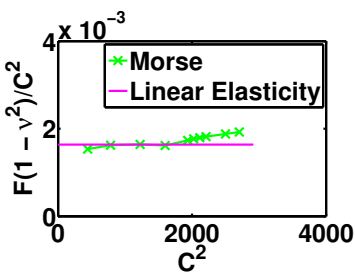

(b)

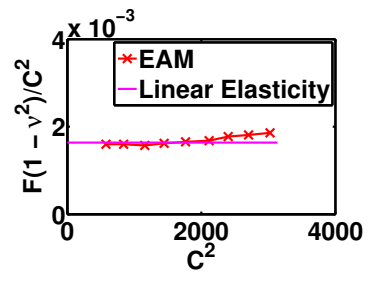

(c)

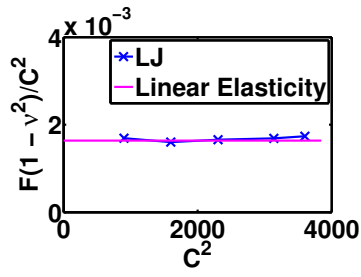

(d)

FIG. 8. Load as a function of square of contact length, $C^{2}$, for different interatomic potentials. The horizontal lines indicate the value of $F\left(1-\nu^{2}\right) / C^{2}$ given by Hertzian contact theory in the limit of infinite L given by $77^{38}$.

\section{SCALING ANALYSIS}

Here, we study the dependence of $\frac{F_{c}}{R}, \frac{C_{c}}{R}, \frac{\xi}{R}$ and $\frac{Y^{*}}{R}$ with $R$. First, we ask how well linear contact mechanics describes $F$ and $C$ vs. $D$ before the bifurcation. The analytical expressions for load and indenter depth for a thick linear elastic isotropic infinite layer being indented by a long cylinder were developed by Meijers 38 . (7) and (8) give load and 
indenter depth respectively as a function of indenter radius, $R$, contact length, $C$, layer thickness, L, and material properties, $E$ (Young's Modulus) \& $\nu$ (Poisson's Ratio). In

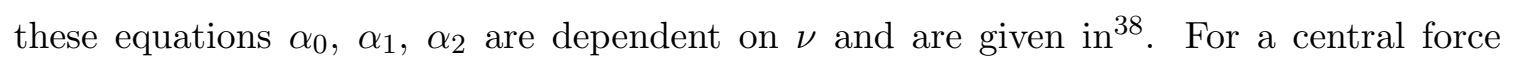
pair potential on a hexagonal lattice, $\nu$ is 0.25 and $\alpha_{0}, \alpha_{1}, \alpha_{2}$ are $-2.27,5.4$ and -7.24 respectively. Note that in (8), $D$ has logarithmic divergence with $L$ in two dimensions.

$$
\begin{gathered}
F=\frac{\pi C^{2} E}{16 R\left(1-\nu^{2}\right)}\left[1+1 / 8 * \alpha_{1}(C / 2 L)^{2}+1 / 64\left(\alpha_{1}^{2}+6 \alpha_{2}\right)(C / 2 L)^{4}\right] \\
D=C^{2} / 4 R\left(\left(1 / 2+1 / 16 \alpha_{1}(C / 2 L)^{2}+1 / 128\left(\alpha_{1}^{2}+6 \alpha_{2}\right)(C / 2 L)^{4}\right) \ln (8 L / C)\right. \\
\left.+1 / 4+1 / 4 \alpha_{0}+1 / 64 \alpha_{1}\left(3+2 \alpha_{0}\right)(C / 2 L)^{2}+1 / 512\left(2 \alpha_{0} \alpha_{1}^{2}+12 \alpha_{0} \alpha_{2}+3 \alpha_{1}^{2}+10 \alpha_{2}\right)(C / 2 L)^{4}\right)
\end{gathered}
$$

In Fig. 7(a), we study the variation of $F$ vs. $D$ for $R=160$, LJ- $O_{1}$. The Hertzian theory gives a good description of load vs. depth with a slight but systematic underestimation of the load just before nucleation. Note that since $D$ has a logarithmic divergence with $L$ in two dimensions, it is more convenient to study $F$ vs. $C$ as there is a well defined infinite $L$ limit, and we plot this in Fig. 7(b). In general, we can expect $L$ independence in the $F$ vs. $C$ curves when $L>>C_{c} / 2$. We show below that for all of our samples, $C_{c} \approx R / 2$, so as long as $L>>R / 4$, we can expect $L$ independence in the $F$ vs. $C$ curves.

For these 2D systems, we define hardness as the ratio of critical load, $F_{c}$, to the indenter radius, $R$. In Fig. 9, we plot the critical load and contact length as a function of indenter radius for various sample sizes. As explained above, within linear contact mechanics, $F$ is determined by $C, R$ and $L$. So as long as we are in the $L>>R$ limit and linear contact mechanics applies, the critical load, $F_{c}$, should be determined by the critical contact length, $C_{c}$, and indenter radius, $\mathrm{R}$. When $R>100$ we observe collapse of hardness, $F_{c} / R$, for various $L$ as shown in Fig. 9(b) for LJ- $O_{1}$. For this range of $R$ as observed in Fig. 9(a), $C_{c}$ varies linearly with $R$, and according to $77 F_{c}$ varies as $C_{c}^{2} / R$. Therefore, hardness also remain independent of $R$ as can be seen in Fig. 9(b). For small indenter radii $(R<100)$, 


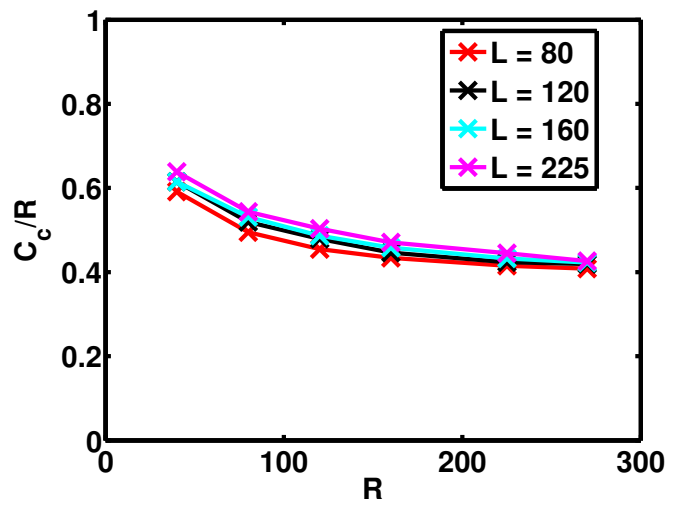

(a)

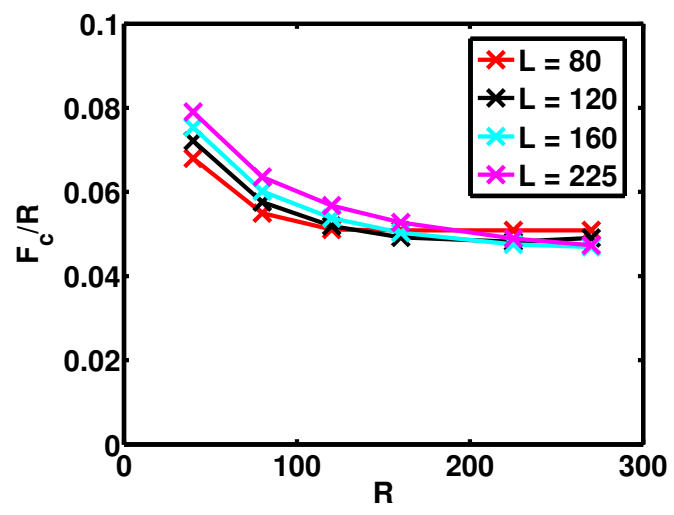

(b)

FIG. 9. (a) Critical contact length, $C_{c}$, as a function of indenter radius, $R$, for various $L, O_{1}$, LJ crystal . (b) Indenter Force, $F_{c}$, in the last stable configuration scaled by indenter radius, $R$, as a function of $R$ for different system sizes, $O_{1}$, LJ crystal.

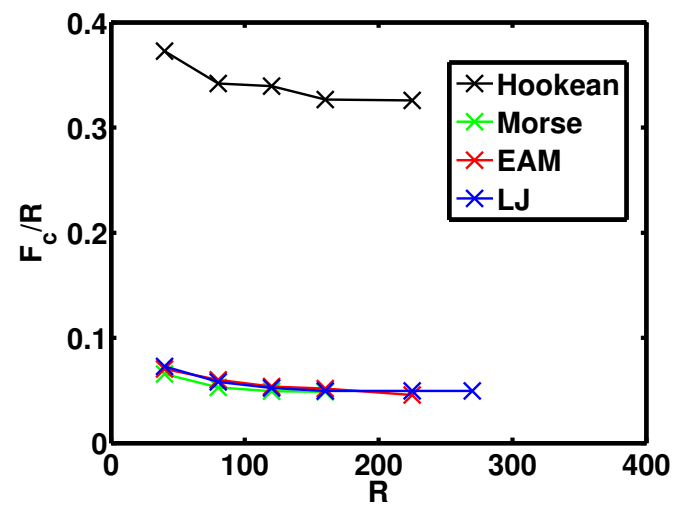

(a)

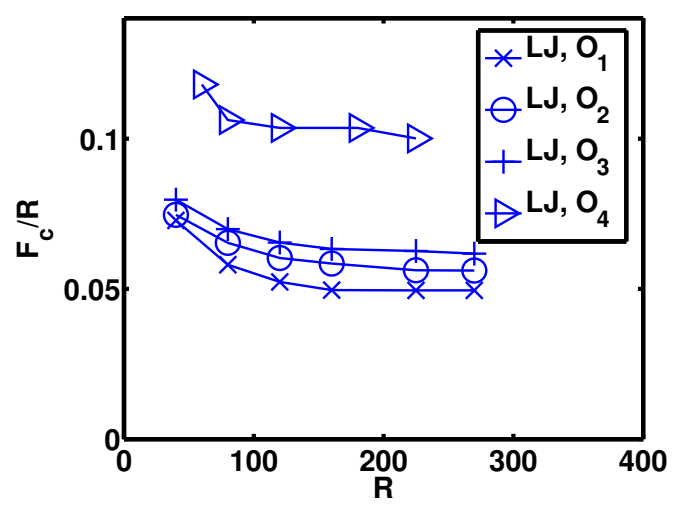

(b)

FIG. 10. Critical force, $F_{c}$, scaled by $R$ as a function of $R$ for $L=160$ (a) various interatomic potentials (b) various orientations.

the crystal is relatively harder and indentation size effect (ISE) is observed in both $C_{c} / R$ and $F_{c} / R$ curves (Fig. 9).

We calculate hardness values for different interatomic potentials and non-dimensionalize 
them by their Young's Modulus, E, calculated for the initial undeformed crystals. The variation of this nondimensional hardness as a function of $R$ for different interatomic potentials and orientations is shown in Fig. 10. The fact that the nondimensional hardness values for different potentials are not equal even though the nondimensionalized $F$ vs. $C$ curves for all the potentials approximately collapse up to nucleation (Fig. 10 and 8), indicates the importance of non-linear behavior of interatomic potentials for determining the prefactors. It is interesting that the harmonic crystal has the highest non-dimensionalized hardness, and thus provides us limiting value estimates of the hardness. Among different orientations, the most stable surface orientation, $O_{1}$, has the lowest hardness value. $O_{4}$, for which the indenter axis is parallel to the crystal axis has roughly twice the hardness of $O_{1}$.

Previous authors $(26,25)$ have employed quasicontinuum simulations for significantly large system size regimes such that their results are independent of $L$. For EAM potential MR observed $F_{c} / C_{c}$ to be between $0.28 \mathrm{ev} / A^{o 3}-0.33 \mathrm{ev} / A^{o 3}$ for the systems that were two order of magnitude bigger than our systems. For our $L=120, R=120$ EAM potential crystal $F_{c} / C_{c}$ is found to be $0.29 \mathrm{ev} / A^{o 3}$ that matches well with MR. Our simulation results clearly show that if $L$ is bigger than 100, the system size effects are no longer important and fully atomistic simulations can yield the required information. For the range of $R$ of interest, we also calculate $C_{c}$ using linear elasticity for known $F_{c}$ and $R . F_{c} / C_{c}$ using this linear elastic calculation is $0.25 \mathrm{ev} / A^{o 3}$ which is roughly $13 \%$ lower than the actual value showing that deviation from linear contact mechanics are non-negligible.

We measure depth of the embryo from the bottom most point of the deformed surface, $Y^{*}$, and the embryo size, $\xi$. Both were also found to be roughly independent of $L$ as shown in Fig. 11 for LJ- $O_{1}$. We observe the following scaling laws for $Y^{*}$ and $\xi$.

$$
Y^{*} \sim R^{0.75}, \xi \sim R^{0.5}
$$

These scaling laws are essentially independent of interatomic potential and crystallographic orientation as shown in Fig. 12. In the limit of large $R \xi / R$ and $Y^{*} / R$ display non-trivial scaling with $R$ despite the $R$ independence of $F_{c} / R$ and $C_{c} / R$. If $R$ is the only relevant 


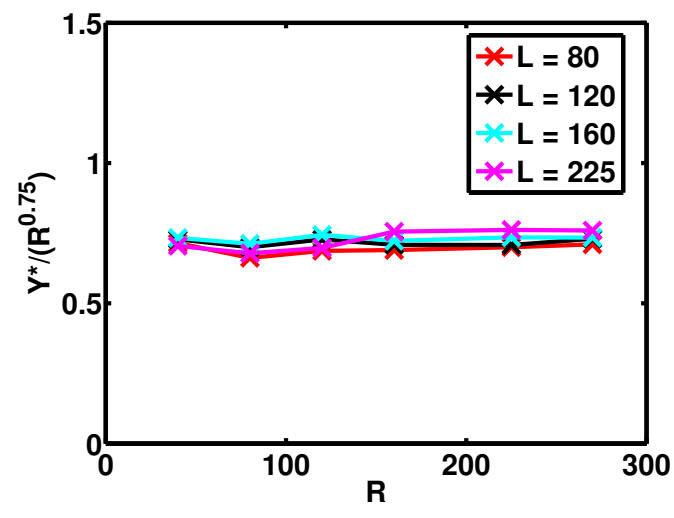

(a)

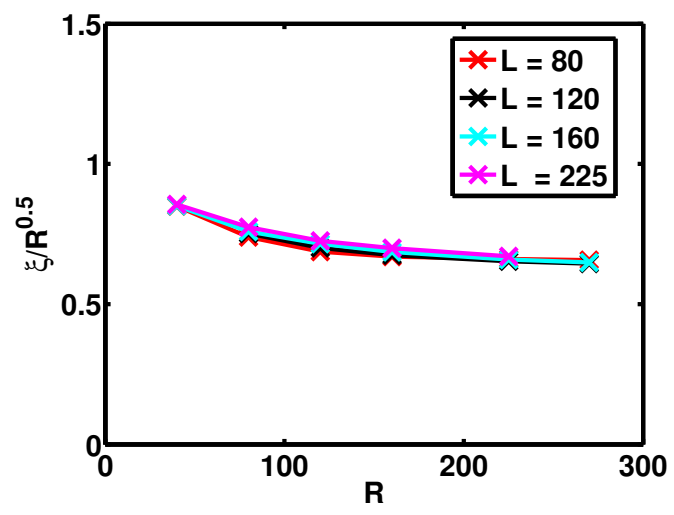

(b)

FIG. 11. (a) Depth of embryo center, $Y^{*}$, scaled by $R^{0.75}$ (b) Embryo size, $\xi$, scaled by $R^{0.5}$ - as a function of $R$ for different system sizes, $O_{1}$, LJ crystal.

length scale, then any linear continuum description cannot explain these scaling laws. This implies that even though the linear elastic calculation is close to the actual $F$ vs. $C$ curves the non-linear behavior of material is important for explaining these non-trivial scaling laws for $\xi / R$ and $Y^{*} / R$.

The exact form of the relation between the infinite size limits of $F c / R$ and $\xi / R^{0.5}$ is not clear, but there is trend that correlates bigger embryos with lower hardness. For instance, LJ, Morse, and EAM all have $F_{c} / R 0.05$ to within a few percent in orientation $O 1$ and have similar embryo size $\xi / R^{0.5}=0.61 \pm 0.05$. The $O_{2}$ and $O_{3}$ LJ orientations have similar $F c / R$ and $\xi / R^{0.5}$ but the $O_{4}$ orientation has a signficantly larger hardness and a slightly smaller $\xi / R^{0.5}$ than the other orientations. So for LJ $O_{4}$, the trend is: "smaller is harder," but not dramatically so. The Hookean system, on the other hand, has a dramatically larger hardness and larger scaled embryo size, $\xi / R^{0.5}$; the opposite trend from LJ $O_{4}$. So, while the scaling law for $\xi$ and the ISE observed in both $F_{c} / R$ and $\xi / R^{0.5}$ are robust across all potentials and orientations, the prefactors which enter those scaling laws may have an independent origin from each other. i.e. for LJ- $O_{4}$ smaller is harder, while for Hookean- $O_{1}$, smaller is softer. 


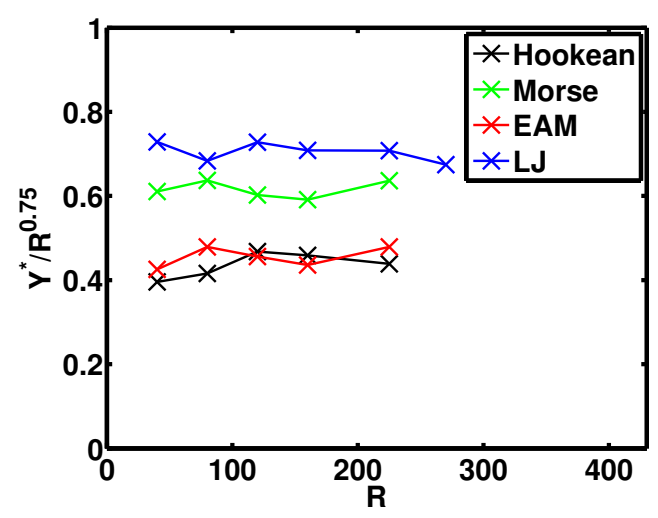

(a)

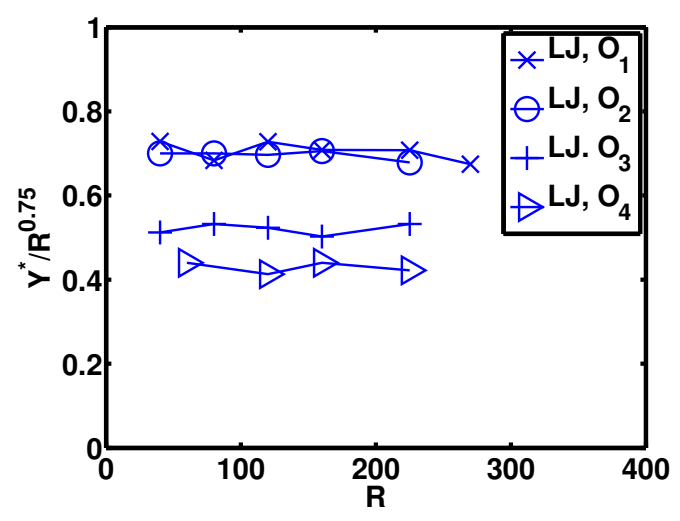

(c)

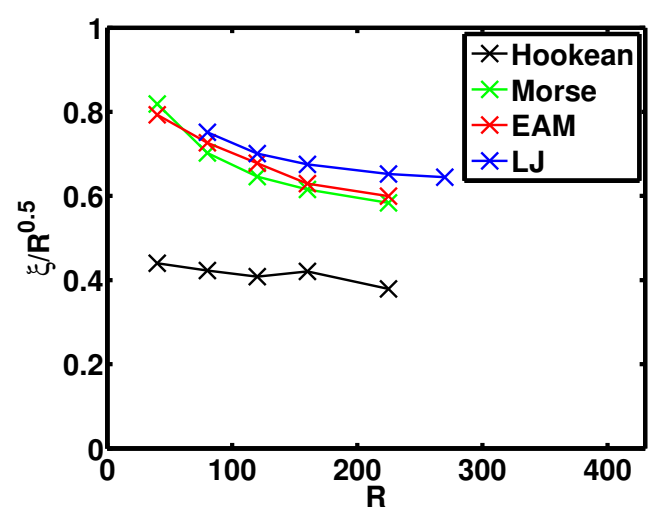

(b)

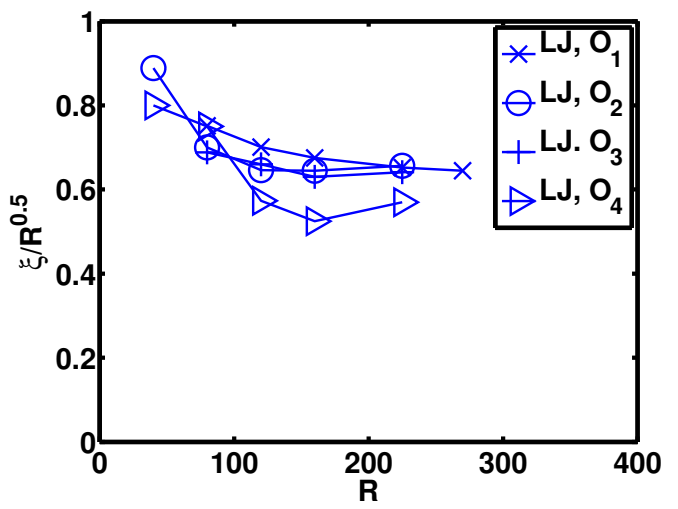

(d)

FIG. 12. (a) Embryo center depth, $Y^{*}$, scaled by $R^{0.75}$ (b) Embryo size, $\xi$, scaled by $R^{0.5}$ - as a function of $R$ for different orientations and interatomic potentials.

\section{V. $\Lambda$ CRITERION}

Another question that has been investigated by many authors before is, whether it is possible to predict the onset of instability for HDN using a criterion? As shown in Fig. 5(a), the instability process involves a cluster of atoms (10 to 20 atoms) and is inherently non-local as described by $\mathrm{MR}^{26}$. Therefore, it is still not fully understood if this instability can be predicted by a locally measurable threshold criterion . The first 
naive local-criterion proposed was based on Maximum Resolved Shear Stress (MRSS) or Schmid factor. The resolved shear stress, $\tau$, can be calculated using the projection, of the component of atomic-level shear stress on the critical plane, along the slip direction. The naive expectation is that the dislocation loop will nucleate at the location of $\tau_{\max }$, when $\tau$ exceeds the source threshold characteristic of the material. The maximum resolved shear stress (MRSS) calculated for LJ Potential, $O_{1}$ and $O_{4}$ is shown in Fig. 13. Clearly, the MRSS does not predict the location of embryo accurately, as already shown by Kelchner ${ }^{\mathbf{9}}$.

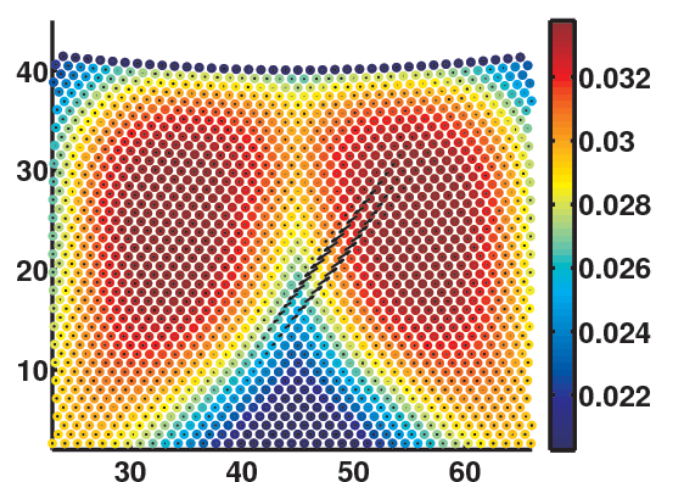

(a)

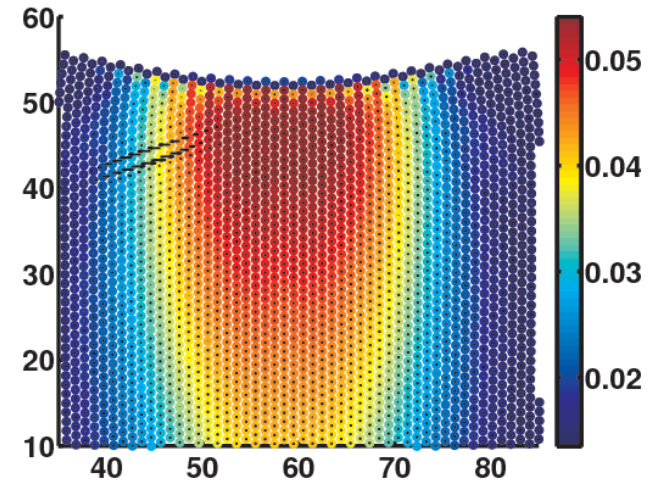

(b)

FIG. 13. Maximum resolved shear stress for each atom in LJ crystal, $O_{1}$ and $O_{4}$.

Later, Li et al. formulated another local criterion, the $\Lambda$ criterion ${ }^{312824}$. $\Lambda$ is based on Hill's analysis of the stability of plane waves in a homogeneously deformed crystal. $\Lambda$ is a function of local strain, which can be computed through several approaches, given the atomic positions in the crystal. We describe here two methodologies for local strain calculation, and for both cases our results are almost the same. One can define the local strain on each triangle in a 2D hexagonal lattice using linear interpolation, as the strain was computed during the calculation of $\Omega$ in section III. For each atom, the local strain is the algebraic average of the strain of six triangles around the atom. We also computed $\Lambda$ for each triangle, instead of each atom, based on the strain in each triangle and the results were also almost the same in this case. The other procedure to calculate local strain is 
as done by Falk and Langer in 39 . In this case, the local strain for an atom is the best fit uniform strain calculated using the nearest neighboring atoms.

Given the local deformation gradient, $F$, at particle, $i$, the initial lattice is homogeneously deformed. The dynamical matrix, $D_{i \mu \nu}$, for the homogeneously deformed lattice at $i^{\text {th }}$ particle can be computed as:

$$
D_{i \mu \nu}(\vec{k})=\sum_{j} H_{\mu \nu}\left(\overrightarrow{R_{i j}}\right) e^{-i \vec{k} \cdot \overrightarrow{R_{i j}}}
$$

For a $2 \mathrm{D}$ hexgonal lattice, $D_{i \mu \nu}$ is a $2 \mathrm{x} 2$ matrix indexed indexed by $\mu, \nu . \overrightarrow{R_{i j}}$ is the displacement vector defined from particle, $i$, to the neighboring particle, $j$, in the homogeneously deformed crystal. $H_{\mu \nu}$ contains the elements of the hessian matrix for a homogeneously deformed crystal. $k$ and $\hat{k}$ are respectively the magnitude and direction of the wavevector $\vec{k}$.

Furthermore, 10 can be simplified as:

$$
D_{i \mu \nu}(\vec{k})=\sum_{j} H_{\mu \nu}\left(\overrightarrow{R_{i j}}\right)\left(\cos \left(k \hat{k} \cdot \overrightarrow{R_{i j}}\right)-1\right)
$$

Then using the long wavelength approximation,

$$
\begin{array}{r}
D_{i \mu \nu}(\vec{k}) \approx \sum_{j}-0.5 H_{\mu \nu}\left(\overrightarrow{R_{i j}}\right)\left(k \hat{k} \cdot \overrightarrow{R_{i j}}\right)^{2} \\
=\left(k^{2}\right) \sum_{j}-0.5 H_{\mu \nu}\left(\overrightarrow{R_{i j}}\right)\left(\hat{k} \cdot \overrightarrow{R_{i j}}\right)^{2} \\
\Lambda_{i \mu \nu}=\sum_{j}-0.5 H_{\mu \nu}\left(\overrightarrow{R_{i j}}\right)\left(\hat{k} \cdot \overrightarrow{R_{i j}}\right)^{2}
\end{array}
$$

For particle $i, \Lambda$ is the minimum eigenvalue of $\Lambda_{i \mu \nu}$ over all $\vec{k} . \Lambda_{i \mu \nu}\{\vec{k}\}$ is the acoustic tensor. It is related to the dynamical matrix which gives the vibrational frequencies of phonons of a given wavevector. $k^{2} \Lambda_{\min }$ is then the lowest squared phonon frequency for the phonon of given wavevector. A negative value of $\Lambda_{\text {min }}$ indicates an unstable phonon mode. 


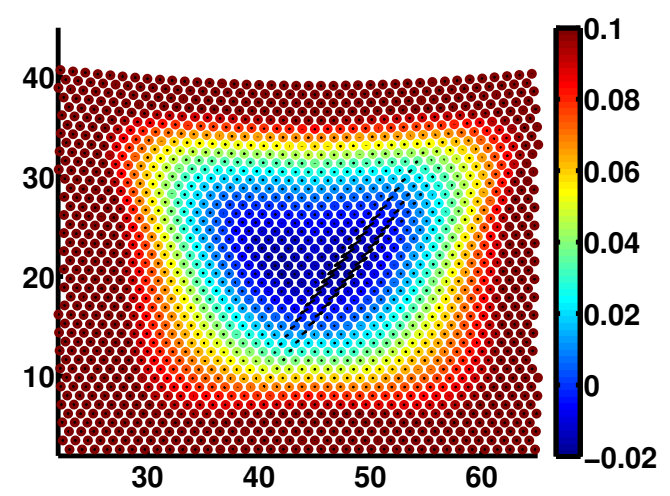

(a) LJ Crystal, $O_{1}$ orientaion

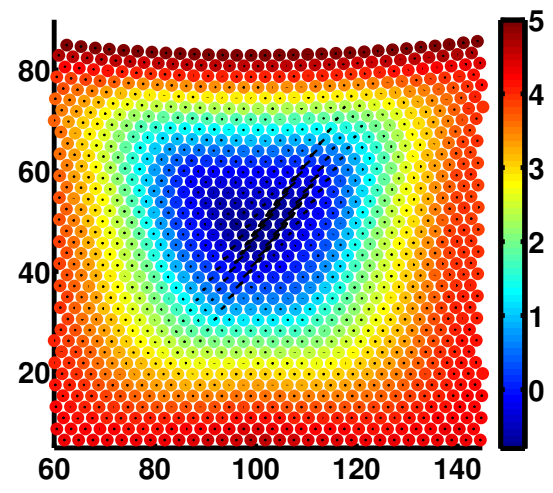

(c) EAM Crystal, $O_{1}$ orientaion

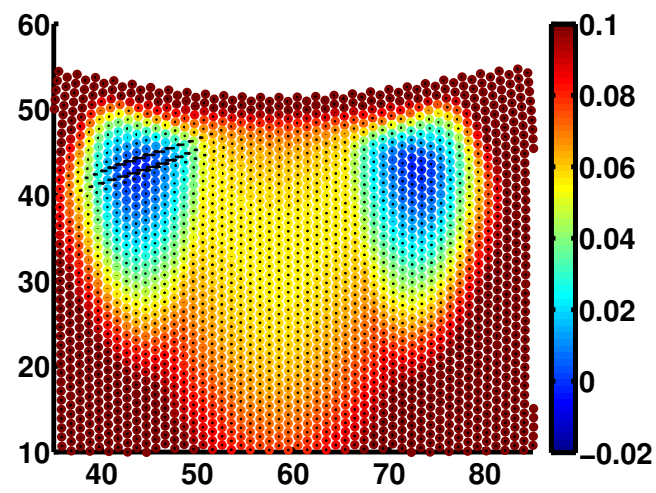

(b) LJ Crystal, $O_{4}$ orientaion

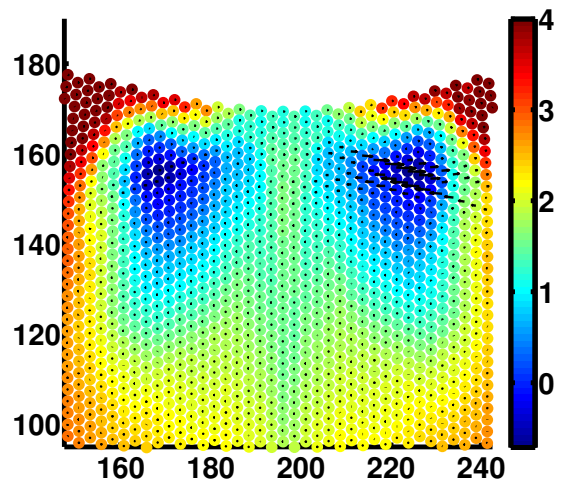

(d) EAM Crystal, $O_{4}$ orientaion

FIG. 14. Minimum $\Lambda$ value for each atom in LJ and EAM crystal, $O_{1}$ and $O_{4}$.

$\Lambda$ for EAM and LJ potentials for the stable surface orientation, $O_{1}$, and the unstable surface orientation, $\mathrm{O}_{4}$, is shown in Fig. 14. Note that $\Lambda$ becomes negative before instability, this makes a strict correspondence between loss of stability and $\Lambda<0$ untenable. Recently, $\mathrm{MR}^{26}$ also showed that $\Lambda$ becomes negative before the actual instability. Furthermore, they observed that $\Lambda_{\min }$ does not correspond to the embryo location for EAM- $O_{4}$. On the contrary, we always observe that $\Lambda$ is minimum at the center of the embryo irrespective of the crystal orientation and the inter-atomic potential.

In Fig. 15 we also show the wave-vector direction, $\hat{k}$, and the polarization direction, 
$\hat{p}$, corresponding to the least stable phonon, for the atom with minimum $\Lambda$. As shown, the wave-vector direction, $\hat{k}$, is almost perpendicular to the slip plane. The eigenvector corresponding to the minimum eigenvalue of $\Lambda_{i u v}$ or the polarization direction, $\hat{p}$, is not perpendicular to the wave-vector direction, $\hat{k}$; it is very close to the slip direction. These results are consistent across all crystal orientations and inter-atomic potentials.

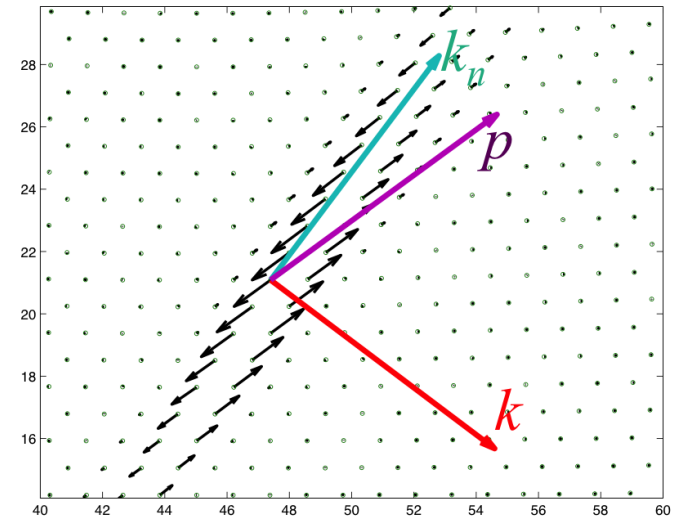

(a) LJ Crystal, $O_{1}$ orientaion

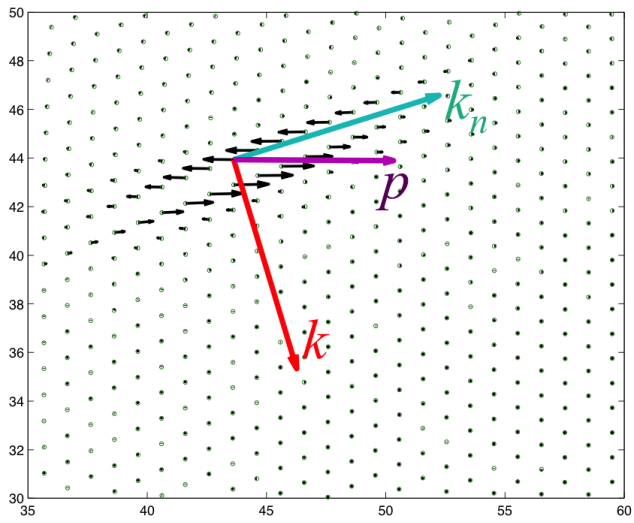

(b) LJ Crystal, $\mathrm{O}_{4}$ orientaion

FIG. 15. Wavevector direction, $\hat{k}$; Normal to wavevector direction, $\hat{k_{n}}$; Polarization vector direction, $\hat{p}$ corresponding to minimum $\Lambda$ in LJ Crystal for (a): $O_{1}$, (b): $O_{4}$.

\section{SUMMARY AND DISCUSSION}

To summarize the key results, we have shown that: i) linear elastic Hertzian contact mechanics works well for all system sizes, crystallographic orientations, and interatomic potentials with a slight but systematic underestimation of the load all the way up to instability; ii) $F_{c} / R$ and $C_{c} / R$ approach well defined asymptotic values for each system in the large $R$ limit, but there is a systematic indentation size effect where both $F_{c} / R$ and $C_{c} / R$ increase at small $R$ and the correspondence between $F_{c}$ and $C_{c}$ can be understood simply as a consequence of the validity of linear elastic contact mechanics; iii) the dislocation embryo grows dramatically with indenter radius in agreement with Miller and Rodney ${ }^{26}$ with an 
asymptotic scaling law of $\xi \sim R^{0.5}$ in the large $R$ limit; iv) $\xi / R^{0.5}$ exhibits the same type of indentation size effect as $F_{c} / R$ and $C_{c} / R\left(\xi / R^{0.5}\right.$ increases at small $\left.R\right)$ for all systems; v) the depth of the embryo, $Y_{*}$, follows another non-trivial scaling law: $Y_{*} \sim R^{0.75}$ vi) the lowest eigenvalue, $\Lambda$, of the locally defined acoustic tensor, although already negative by the time dislocations nucleate, is always smallest (most negative) at the center of the dislocation embryo and the polarization of the most unstable phonon gives precise agreement with the local direction of the unstable mode.

For the scaling of the embryo size, a local atomistic phenomenon would imply $\xi \sim R^{0}$, while a scale free continuum description would imply $\xi \sim R^{1}$. The exponent of $1 / 2$ is intriguing and it will be interesting in the future to see if a simple continuum theory incorporating stress gradients ${ }^{32}$ or a more elaborate scheme such as a non-local extension of the Peierls-Nabarro-Rice mode ${ }^{19 \mid 20}$ could naturally capture the scaling behavior of the embryo size.

There is an important paradox lurking in our results. First, we have argued that linear elastic contact mechanics gives a good description of the loading curves right up to instability. Then, we have shown that the location of the embryo has non-trivial scaling: $Y_{*} \sim R^{0.75}$. One might say, from the continuum point of view, that the embryo recedes to the surface as the indenter grows. Finally, we have shown that the $\Lambda$ criterion correctly predicts the location of the embryo. But the only input into the $\Lambda$ calculation is the local atomic strain. So if linear contact mechanics gave the correct strain field, then the spatial structure of the $\Lambda$ field could only have trivial scaling with $R$ and could never give $Y_{*} \sim R^{0.75}$.

Resolving this paradox may lead the way to constructing the appropriate framework (such as a stress gradient, or non-local-Peierls approach) to understand the scaling of the embryo size and could allow for understanding the entire nucleation problem. We suspect that the simplest resolution to the paradox is that while Hertzian contact theory does a good job predicting the load and contact length, it does a poorer job describing the 
sub-surface strain fields. Preliminary results support this conjecture.

We have also shown that there is a true indentation size effect of the "smaller is harder" variety for all systems. The embryo size has a similar trend: at small $R$, both $F_{c} / R$ and $\xi / R^{0.5}$ increase significantly above their large $R$ asymptotic values. One might be tempted to conclude that "smaller is harder and smaller is longer; so longer is harder". If we compare different orientations and interaction potentials, it turns out that this is not the case. On one hand, the Hookean lattice is by far the hardest of all, and it obeys the "longer is harder" trend. The LJ, Morse, and EAM potentials in orientation $O 1$ all had roughly the same dimensionless hardness, $F_{c} / R \approx 0.05$, while the Hookean had $F_{c} / R \approx 0.33$, almost a factor of 7 greater than the others. On the other hand, if one looks at the asymptotic $F_{c} / R$ and $\xi / R^{0.5}$ values for the different orientations of the LJ crystal, there is no clear correlation between harder orientations and longer embryos.

This work and other recent simulations and theoretical analysis of indentation nucleation have focused largely on geometrical properties of the embryo - primarily its location. However, what is more relevant for connecting to experiments is the energy barrier (or barrier $s$ if there are other nearby low-lying transition paths made accessibly by the loading conditions). Recent experimental studies have looked at the distribution of time-to-first pop-in or depth-to-first pop-in at various temperature and loading rate $17 / 40$. We have shown above that the bifurcation is of saddle-node type. In very general contexts 4142 , saddle node bifurcations lead to non-Eyring transition rates in the vicinity of the bifurcation. Johnson and Samwer have argued ${ }^{42}$ that this gives rise to a universal scaling of the reduction of the flow stress with temperature and shear rate in metallic glasses. A similar theory could be developed for the distribution of pop-in events at various temperature and loading rate. The key parameter in such a theory would be the pre-factor in the $\lambda \sim \sqrt{\delta D}$ scaling law (the offset of the cyan line in Fig. 44. Determining how this pre-factor depends on $R$ and whether it is related to the non-trivial scaling behavior of $\xi$ would be crucial in formulating a statistical theory of thermally activated pop-in events. 
Finally, we point out that all of the results presented here are for $2 D$. Studies are currently underway for f.c.c. metals, and indicate that the scaling for the embryo length in $2 D$ carries over precisely to the embryonic loop radius in f.c.c. metals. Since the results for the scaling of $\xi$ with $R$ seem so robust, it is obvious to ask when they break down. So studying other lattices (e.g. b.c.c. or h.c.p. metals) and covalently bonded systems will be important future work.

$$
P_{f r}\left(v, \sigma_{I}, V_{f}\right)=1-\exp \left[-\frac{v\left(V_{f}\right)^{n}}{v_{0}}\left(\frac{\sigma_{I}}{\sigma_{w}}\right)^{m}\right]
$$

\section{ACKNOWLEDGEMENTS}

We thank Amit Acharya for useful discussions at various stages during this work. This material is based upon the work supported by the National Science Foundation under Award Number CMMI-1100245.

1 E. Rabkin, H. S. Nam, and D. J. Srolovitz, ACTA MATERIALIA 55, 2085 (2007).

2 E. Rabkin and D. J. Srolovitz, NANO LETTERS 7, 101 (2007).

3 T. Zhu, J. Li, A. Samanta, A. Leach, and K. Gall, PHYSICAL REVIEW LETTERS 100, $25502(2008)$.

4 J. R. Greer, C. R. Weinberger, and W. Cai, MATERIALS SCIENCE AND ENGINEERING A-STRUCTURAL MATERIALS PROPERTIES MICROSTRUCTURE AND PROCESSING 493, 21 (2008).

5 D. Mordehai, M. Kazakevich, D. J. Srolovitz, and E. Rabkin, ACTA MATERIALIA 59, 2309 (2011).

${ }^{6}$ D. Mordehai, S. W. Lee, B. Backes, D. J. Srolovitz, W. D. Nix, and E. Rabkin, ACTA MATERIALIA 59, 5202 (2011).

7 W. W. Gerberich, J. C. Nelson, E. T. Lilleodden, P. Anderson, and J. T. Wyrobek, ACTA MATERIALIA 44, 3585 (1996). 
8 S. G. Corcoran, R. J. Colton, E. T. Lilleodden, and W. W. Gerberich, Physical Review B 55, R16057 (1997).

9 C. L. Kelchner, S. J. Plimpton, and J. C. Hamilton, Physical Review B 58, 11085 (1998),

10 E. B. Tadmor, R. Miller, R. Phillips, and M. Ortiz, JOURNAL OF MATERIALS RESEARCH 14, $2233(1999)$

11 J. A. Zimmerman, C. L. Kelchner, P. A. Klein, J. C. Hamilton, and S. M. Foiles, PHYSICAL REVIEW LETTERS 87, art. no. (2001).

12 E. T. Lilleodden, J. A. Zimmerman, S. M. Foiles, and W. D. Nix, JOURNAL OF THE MECHANICS AND PHYSICS OF SOLIDS 51, 901 (2003)

13 K. J. Van Vliet, J. Li, T. Zhu, S. Yip, and S. Suresh, PHYSICAL REVIEW B 67, 104105 (2003)

14 J. K. Mason, A. C. Lund, and C. A. Schuh, PHYSICAL REVIEW B 73, 54102 (2006).

15 P. Schall, I. Cohen, D. A. Weitz, and F. Spaepen, Nature 440, 319 (2006).

16 R. J. Wagner, L. Ma, F. Tavazza, and L. E. Levine, Journal of Applied Physics 104, 114311 (2008)

17 J. R. Morris, H. Bei, G. M. Pharr, and E. P. George, PHYSICAL REVIEW LETTERS 106, $165502(2011)$.

18 V. B. Shenoy, R. Phillips, and E. B. Tadmor, Journal of the Mechanics and Physics of Solids 48, $649(2000)$.

19 J. R. Rice, Journal of the Mechanics and Physics of Solids 40, 239 (1992).

20 J. R. Rice and R. Thomson, Philosophical Magazine 29,43 (1974), http://dx.doi.org/10.1080/14786437408213555

21 O. Rodrguez de la Fuente, J. A. Zimmerman, M. A. Gonzlez, J. de la Figuera, J. C. Hamilton, W. W. Pai, and J. M. Rojo, Physical Review Letters 88, 036101 (2002).

22 A. Gouldstone, K. J. Van Vliet, and S. Suresh, Nature 411, 656 (2001).

23 K. Van Vliet, J. Li, T. Zhu, S. Yip, and S. Suresh, Physical Review B 67 (2003), 10.1103/PhysRevB.67.104105.

24 T. Zhu, J. Li, K. J. Van Vliet, S. Ogata, S. Yip, and S. Suresh, Journal of the Mechanics and Physics of Solids 52, 691 (2004).

25 J. Li, T. Zhu, S. Yip, K. J. Van Vliet, and S. Suresh, Materials Science and Engineering: A 365, 25 (2004). 
26 R. Miller and D. Rodney, Journal of the Mechanics and Physics of Solids 56, 1203 (2008).

27 In all cases, we work in a regime where the results are effectively independent of the sample size and should be directly comparable to the results of Miller and Rodney 26 .

28 T. Zhu, J. Li, and S. Yip, Physical Review Letters 93 (2004), 10.1103/PhysRevLett.93.025503.

29 S. Plimpton, Journal of Computational Physics 117, 1 (1995).

30 E. Jones, T. Oliphant, P. Peterson, et al., (2001-), [Online; accessed 2014-08-28].

31 J. Li, K. J. Van Vliet, T. Zhu, S. Yip, and S. Suresh, Nature 418, 307 (2002).

32 R. E. Miller and A. Acharya, JOURNAL OF THE MECHANICS AND PHYSICS OF SOLIDS 52, 15071525 (2004).

33 F. Ercolessi and J. B. Adams, MRS Online Proceedings Library 291, null (1992).

34 A. Hasan, International Journal for Multiscale Computational Engineering (2011), 10.1615/IntJMultCompEng.2011002554.

35 A. Garg, A. Acharya, and C. Maloney, Journal of the Mechanics and Physics of Solids , 76 (2015).

36 A. Lematre and C. Maloney, Journal of Statistical Physics 123, 415 (2006).

37 J. Zimmerman, C. Kelchner, P. Klein, J. Hamilton, and S. Foiles, Physical Review Letters 87 (2001), 10.1103/PhysRevLett.87.165507.

38 P. Meijers, Applied Scientific Research 18, 353 (1968).

39 M. L. Falk and J. S. Langer, Physical Review E 57, 7192 (1998), arXiv:cond-mat/9712114.

40 J. K. Mason, A. C. Lund, and C. A. Schuh, PHYSICAL REVIEW B 73, 054102.

41 C. Maloney and A. Lematre, Physical Review E 74 (2006), 10.1103/PhysRevE.74.016118.

42 W. L. Johnson and K. Samwer, Phys. Rev. Lett. 95, 195501 (2005). 